\title{
Science sociale et polyphonie
}

Réflexions sur le triangle sociologie, communication, action

Social Science and Polyphony. Reflections about Sociology, Communication, Action

\section{Mathieu Quet}

\section{OpenEdition \\ Journals}

Édition électronique

URL : http://journals.openedition.org/questionsdecommunication/1249

DOI : 10.4000 /questionsdecommunication. 1249

ISSN : 2259-8901

\section{Éditeur}

Presses universitaires de Lorraine

\section{Édition imprimée}

Date de publication : 1 décembre 2008

Pagination : 283-306

ISBN : 978-2-86480-981-4

ISSN : $1633-5961$

Référence électronique

Mathieu Quet, «Science sociale et polyphonie », Questions de communication [En ligne], 14 | 2008, mis en ligne le 19 janvier 2012, consulté le 01 mai 2019. URL : http://journals.openedition.org/ questionsdecommunication/1249; DOI : 10.4000/questionsdecommunication.1249 


\title{
$>$ NOTES DE RECHERCHE
}

\author{
MATHIEU QUET \\ Centre Koyré \\ École des hautes études en sciences sociales, Paris \\ matthieuq@free.fr
}

\section{SCIENCE SOCIALE ET POLYPHONIE. RÉFLEXIONS SUR LE TRIANGLE SOCIOLOGIE, COMMUNICATION, ACTION}

\begin{abstract}
Résumé. — La sociologie de tradition durkheimienne soulève un problème d'ordre communicationnel par la performativité qu'elle prête au discours sociologique. En effet, la sociologie durkheimienne s'est construite sur la fiction d'un discours rendu actif par sa rationalité et sa vérité, et c'est l'élaboration de cette fiction qui est ici analysée à travers des textes de Condorcet, Comte et Durkheim. Celleci est problématique car elle ne tient pas compte de l'indépassable polyphonie des discours. La seconde partie de la contribution revient donc sur la notion de polyphonie des discours, en montrant les prétentions du discours sociologique à mettre fin à cette polyphonie, malgré l'impossibilité d'un tel geste. L'article se clôt sur un appel à privilégier une analyse politique de la circulation des savoirs et des discours, et à reproblématiser la question de la diffusion des connaissances dans la « société du savoir ».
\end{abstract}

Mots clés. - Polyphonie, performativité, médiations, épistémologie, histoire des sciences sociales, circulation sociale des savoirs. 
$\mathrm{D}$ ans L'esprit sociologique, le sociologue Bernard Lahire (2005) s'inscrit dans la filiation de Pierre Bourdieu et explicite sa conception épistémologique de la discipline. La richesse de son ouvrage nécessiterait une discussion plus approfondie que les quelques remarques ici posées. Pour notre propos, nous nous sommes attardé sur un court extrait du texte qui permet de mettre en lumière un aspect essentiel d'un large courant de la sociologie : il s'agit du quatorzième chapitre : «Vers une utopie réaliste : enseigner les sciences du monde social dès l'école primaire »(Lahire, 2005 : 388-402). Bernard Lahire y promeut l'enseignement des sciences du social dès l'école primaire, comme pilier de la démocratie contemporaine, et y justifie la nécessité de mettre en circulation le savoir sociologique. Ce texte ne doit pas être considéré comme représentatif de l'ensemble du champ sociologique : nombreuses sont les écoles rivales et les postures concurrentes en sociologie et, au sein d'une même école ou tradition de recherche, des schèmes d'intelligibilité différents peuvent se superposer (Berthelot, 1998). II n'en est pas moins exemplaire, car il s'inscrit dans un courant qui, sous la figure tutélaire de Durkheim, parcourt l'histoire de la sociologie française et l'a longtemps dominée.

Une rapide étude de cet extrait permet d'en présenter les principales caractéristiques. Deux idées forces s'en dégagent par lesquelles Bernard Lahire attribue deux propriétés essentielles au discours sociologique.Tout d'abord, le savoir sociologique est présenté comme un outil cognitif et discursif permettant de lutter contre des idéologies, des savoirs illusoires, des discours d'institutions traditionnelles (famille, religion, morale, etc.) et contre tous les discours des « sophistes » en général. Ainsi la diffusion « rationnelle »du savoir sociologique donnerait-elle aux acteurs les moyens de contester les discours d'illusion :

\begin{abstract}
« Le rôle des spécialistes de la communication politique (mais il faudrait plutôt parler de "manipulation politique") ou du marketing, des publicistes, des demisavants, des rhéteurs plus ou moins habiles, bref, de tous les sophistes des temps modernes, n'a cessé de croître, et il serait urgent de transmettre, le plus rationnellement possible et auprès du plus grand nombre, les moyens de déchiffrer et de contester les discours d'illusion tenus sur le monde social. [...] Pourquoi ne pas enseigner les outils et les manières de penser que les sciences sociales ont constitués efficacement depuis plus de cent ans plutôt que de laisser les futurs citoyens construire (ou pas) leur savoir sur le monde social au sein de leur structure familiale ou dans les cadres traditionnels de la socialisation (enseignement religieux, socialisation politique et syndicale, etc.) ? 》 (Lahire, 2005 : 395).
\end{abstract}

La seconde idée consiste en une analogie entre science exacte et science sociale, dont les conséquences sont soulignées. La science donne du recul sur le monde et confère une certaine maîtrise de soi-même comme de l'environnement. La science sociale permet une maitrise similaire du monde social, elle est un outil pour le comportement et la pratique des acteurs : 
«Alors que nous sommes désormais capables d'enseigner l'attitude scientifique à l'égard du monde physique et naturel, nous laissons tranquillement se développer des dispositions magiques et pré-rationnelles vis-à-vis du monde social. Norbert Elias a bien montré que, au cours de l'histoire, les hommes ont progressivement conquis une attitude de distanciation, tout d'abord par rapport aux phénomènes naturels puis, plus difficilement, à l'égard des phénomènes sociaux. En effet, les hommes des sociétés pré-scientifiques ont été matériellement et cognitivement impuissants face aux « caprices de la nature ». La science, elle, s'inscrit dans un processus de distanciation et de contrôle des affects et, par conséquent, dans un processus de civilisation. [...] Mais ce que les hommes ont réussi vis-à-vis de la nature avec force et efficacité, ils n'ont toujours pas su complètement le rééditer concernant le monde social » (Lahire, 2005 : 395-396).

Par la première idée, Bernard Lahire restreint la confrontation de savoirs à un plan discursif : la sociologie permet de démanteler l'argumentation des autres discours sur le social, et montre leur inanité. Certes, les sociologues ne justifient pas la puissance du discours sociologique par une propriété purement discursive, mais par son rapport aux « faits 》: les discours idéologiques ou les savoirs de socialisation traditionnelle seraient susceptibles d'être disqualifiés par le savoir sociologique, car ce dernier est plus résistant aux faits. Mais les « faits » remplissent ici une fonction de légitimation dans un dispositif discursif : le lien qu'ils entretiennent avec le savoir sociologique valide ce dernier en tant que discours, et l'habilite à dénoncer des ensembles discursifs obéissant à des règles de formation différentes. La sociologie dispose ainsi d'un privilège épistémique sur les autres savoirs/discours. La seconde idée (parallèle avec les sciences exactes) entraîne des conséquences pratiques : le savoir des sciences sociales permet d'accéder à la maîtrise de soi-même et de son environnement. II entraîne donc une modification de l'agir, qu'on peut nommer « rationalisation du comportement ».

Nous ne souhaitons contester ni le privilège épistémique de la science sociale, ni la possibilité donnée à un individu, à partir du savoir sociologique, de rationaliser son comportement, et en cela notre analyse n'a rien d'une opposition au modèle de la sociologie holiste. Nous pensons cependant que la conception simultanée de ces deux propriétés du discours sociologique soulève un problème d'ordre communicationnel. En effet, Bernard Lahire ne tient pas compte du fait que tout discours est rendu 《 résistant » ou « stable » par des pratiques qui le relayent. Cela est vrai d'une théorie scientifique qui s'appuie sur un ensemble de pratiques expérimentales et, comme il l'affirme, par la construction d'un certain rapport avec les faits. Mais cela est aussi vrai, malgré toutes leurs différences, des autres discours: un discours idéologique, par exemple, est rendu résistant par l'ensemble des pratiques sociales, économiques, politiques, qui le soutiennent. C'est dans les opérations de relais entre discours et pratiques qu'il faut donc chercher la robustesse des discours, et le rapport du discours sociologique avec les faits n'est qu'un cas particulier de ce 
vaste ensemble. Prendre en considération le lien entre savoirs et pratiques conduit à envisager différemment la diffusion du savoir sociologique : le problème n'est plus de faire reconnaître le bien-fondé d'un savoir ou la dimension idéologique d'un autre, ou de faire adopter « naturellement » des pratiques qui correspondent au savoir le plus en accord avec les faits. II faut déplacer l'interrogation des mécanismes de production du savoir aux mécanismes de sa diffusion et de son appropriation. Le nouveau problème est la modification des pratiques elles-mêmes, phénomène qui ne saurait se suffire de l'enseignement des savoirs adéquats. Nous essayons donc, dans cette contribution, de revenir sur une caractéristique importante et peu questionnée du discours sociologique : sa dimension communicationnelle et, à travers elle, son rapport à la pratique!

Pour cela, nous soumettons l'hypothèse qui suit : un large courant de la sociologie, dans lequel s'inscrit le travail de Bernard Lahire, articule une double ambition : produire un discours doté d'un privilège épistémique, dire du monde quelque chose de plus que les autres formations discursives, de façon à les discréditer sur un plan discursif (en produisant un discours plus « vrai », une théorie plus robuste) ; inscrire ce discours dans les pratiques sociales afin de les rationaliser, régler les pratiques sociopolitiques ${ }^{2}$. Cette double ambition s'exprime par le rapport entre deux mouvements : mouvement de clôture épistémique (établissement de la supériorité du savoir sociologique), qui fonderait un mouvement d'ouverture pratique (adhésion « naturelle »-car rationnelle - au savoir sociologique entraînant une modification des pratiques).

Ce rapport (le mouvement discursif fonde le mouvement pratique) est le cœur du dispositif que nous allons étudier : il renvoie à une fiction communicationnelle selon laquelle la clôture épistémique concerne un plan purement cognitif et discursif,élargi ensuite seulement aux opérations matérielles, par le mouvement d'ouverture pratique. La conception de la diffusion du savoir sociologique qui en résulte repose sur l'image d'un décalage : le savoir sociologique disqualifierait les discours erronés, et provoquerait en conséquence une adhésion rationnelle et une modification des comportements. Or, on ne peut distinguer le plan discursif du plan

\footnotetext{
I Par la même occasion, il s'agit de montrer que les sciences de l'information et de la communication ne se définissent pas seulement par des objets particuliers (media, nouvelles technologies, etc.), mais aussi par des problèmes épistémologiques d'importance, 2 peu ou pas traités jusqu'à présent par les autres sciences sociales.

Nous nous permettons de souligner à nouveau que, contrairement à des théoriciens de la microsociologie comme J.-Cl. Kaufmann (200 I), Fr. de Singly (2005), ou du « réseau » comme Br. Latour (2006), nous ne contestons pas cette double ambition qui nous semble nécessaire à la pratique sociologique. Nous cherchons néanmoins à comprendre les questions qu'elle soulève et les problèmes qu'elle pose en termes communicationnels.
} 
pratique, ou leur attribuer des « temps » différents, car ils sont sans cesse mêlés. S'il est impossible de disqualifier des discours idéologiques par une simple confrontation à des discours plus scientifiques, c'est parce qu'ils relaient des pratiques, et sont relayés par elles. Penser que le discours sociologique peut s'inscrire dans les pratiques simplement parce qu'il est plus « vrai » que les autres et après qu'il les ait disqualifiés, c'est faire l'erreur de croire que des opérations indissolublement discursives et matérielles de médiation, de transmission et d'appropriation des savoirs ne jouent aucun rôle dans leur construction. C'est ignorer l'inscription du discours sociologique dans une polyphonie pour mieux prétendre résoudre cette polyphonie ${ }^{3}$.

Revenir sur l'inscription polyphonique du discours sociologique, c'est alors s'interroger sur les rapports entretenus par le discours sur la société avec l'irréductible pluralité des discours sociaux. Trois aspects importants de la notion de polyphonie la définiront dans un premier temps. Tout d'abord, la société est tissée d'une infinité de discours qui coexistent, se croisent, se rencontrent, s'affrontent parfois. Ce tissage forme une polyphonie à laquelle rien ne peut mettre un terme. La notion de polyphonie permet donc en premier lieu de pointer une caractéristique du monde social : monde qui existe à travers des énoncés et des discours dont la pluralité est toujours renouvelée. La deuxième caractéristique de la notion est qu'elle invite à penser ce monde d'énoncés à la manière d'un espace - sonore et vibratoire -, fait de circulation et d'interférences entre les discours. Enfin, une troisième caractéristique de la polyphonie, sur laquelle ce texte revient plus précisément, est la tension entre le principe de désorganisation des discours qu'elle souligne et les stratégies de réduction de la polyphonie qui lui sont inévitablement opposées. En d'autres termes, penser la polyphonie implique de concevoir la tension entre la disparité des discours et leur tendance à la coagulation, entre hétérogénéité et homogénéisation. De multiples pratiques et stratégies tentent en effet de réduire cette polyphonie en rassemblant des discours, en cherchant à emporter l'adhésion ou en disqualifiant des énoncés concurrents. Cette contribution analyse l'une des stratégies forgées par la sociologie pour résoudre la polyphonie, à travers un dispositif spécifique de production et d'inscription du savoir dans le social ${ }^{4}$.

Nous retracerons d'abord l'élaboration de la tension entre clôture épistémique et ouverture pratique, en abordant trois auteurs de la

3 D'ailleurs, B. Lahire (2002) se montre conscient de ce problème et de la tâche immense qu'il représente, dans un texte qui s'arrête à son seuil.

${ }^{4}$ La polyphonie ainsi conçue n'entretient qu'un rapport terminologique avec la polyphonie. 
sociologie naissante : Condorcet, Comte et Durkheim. Nous essaierons de comprendre comment le discours sociologique a progressivement assumé et pris en charge cette dualité, et comment celle-ci a adopté une forme paradigmatique. Dans un second temps, nous montrerons en quoi cette tension conduit à méconnaître l'existence d'une polyphonie sociale, et des mécanismes par lesquels le savoir sociologique s'inscrit dans les pratiques sociales.

\section{La sociologie entre clôture épistémique et ouverture pratique}

Dans la généalogie habituellement retenue de la sociologie, la période de la Révolution française est considérée comme un tournant majeur. Certaines chronologies inscrivent cette naissance dans un plus long terme, et recensent les conditions d'apparition de cette formation discursive particulière (Heilbron, 1990). On retiendra ici l'importance du lien des sciences sociales avec le projet de maîtrise scientifique du monde naturel, qui les précède. En effet, les XVII et $\mathrm{XVIII}$ e siècles assistent à une reconfiguration complète du monde savant : apparition de l'expérience, formation d'académies, rationalisation des pratiques, etc. (Licoppe, 1996). De cette reconfiguration émerge un projet de maîtrise du monde naturel. Avec l'apparition des sciences du social à la fin du XVIII siècle, on assiste à la fois à un glissement et à un accomplissement de ce projet. Glissement, car le projet de maîtrise du monde naturel devient projet de maitrise du monde social ; accomplissement, car Bacon, Boyle ou Hobbes plaçaient déjà des enjeux politiques dans la science (Shapin, Schaffer, 1989). Lorsque la société devient un objet pour la science (Wagner, 2000), les sciences sociales contiennent l'idée d'une maîtrise du social. La sociologie ordonne : elle tente de comprendre le fonctionnement social, de mettre de l'ordre dans la société, et a une prétention à exercer un pouvoir sur les individus. Le projet sociologique tente de mettre en place un dispositif de production simultanée du savoir et du pouvoir qui s'articule sur le rapport déjà évoqué entre « clôture épistémique » et « ouverture pratique ». Nous allons maintenant voir comment ce rapport s'est construit dans les textes de Condorcet, Comte, et Durkheim5.

\footnotetext{
5 Notre approche de ces auteurs n'a aucune prétention à l'exhaustivité. Disons plutôt que nous utilisons certains de leurs textes pour illustrer notre intuition initiale, que seule une analyse approfondie pourrait consolider.
} 


\section{Condorcet : savoir libérateur ou savoir organisateur?}

Condorcet, plutôt qu'un fondateur de la science sociale, est un fondateur du discours de/sur la science sociale. Sans élaborer de façon systématique des théories sur la société, il tient un discours sur ce que devraient être ces théories. On ne peut donc pas encore parler de science du social, encore moins de sociologie, mais il n'en est pas moins un auteur capital dans la constitution du projet sociologique, par sa foi dans les vertus des Lumières et par sa conception d'un projet républicain alliant étroitement égalité et savoir. Dans Cinq mémoires sur l'instruction publique (|79|), il explique toute l'importance revêtue par une instruction publique qui aurait pour fonction de diffuser des connaissances utiles à la société. On y trouve une conception du rôle du savoir, et en particulier du savoir sur la société, dans l'organisation de celle-ci, qui donne certaines pistes pour aborder notre question. Condorcet fait de la production et de la diffusion de la vérité l'élément essentiel des mécanismes de pouvoir. D'une part, la vérité rend les individus plus autonomes, et leur permet de se protéger des pouvoirs injustes : le savoir a des conséquences importantes au niveau individuel. D'autre part, la vérité exerce elle-même un pouvoir sur les individus qui, à travers elle, accèdent à une sorte d'autocontrôle.

La vérité est liée au pouvoir juste, et il existe une alchimie entre raisonnement juste, gouvernement équitable et liberté : «Plus les hommes sont disposés par éducation à raisonner juste, à saisir les vérités qu'on leur présente, à rejeter les erreurs dont on veut les rendre victimes, plus aussi une nation qui verrait ainsi les lumières s'accroitre de plus en plus, et se répandre sur un plus grand nombre d'individus, doit espérer d'obtenir et de conserver de bonnes lois, une administration sage et une constitution vraiment libre » (Condorcet, |79| : 64). L'autonomie des citoyens est préservée par un régime de vérité. II faut donc donner tous les moyens possibles d'accéder à des connaissances, et permettre aux plus faibles d'acquérir les compétences qui les préserveront d'un pouvoir injuste. Dans une telle situation, des inégalités de savoir seront inévitables, mais n'entraîneront pas de rapports de dépendance, car il faut considérer la nature positive du savoir. Contrairement au mensonge ou au savoir faux, il est un appui pour les plus démunis, non une source de dépendance. Du moins Condorcet (179| : 64-65) place-t-il une grande confiance dans les vertus des connaissances scientifiques, et dans la noblesse d'âme de ceux qui les enseignent:

« C'est $[\ldots]$ un devoir de la société que d'offrir à tous les moyens d'acquérir les connaissances auxquelles la force de leur intelligence et le temps qu'ils peuvent employer à s'instruire leur permettent d'atteindre. II en résultera sans doute une différence plus grande en faveur de ceux qui ont plus de talent naturel, et à qui une fortune indépendante laisse la liberté de consacrer plus d'années à l'étude ; mais si cette inégalité ne soumet pas un homme à un autre, si elle offre un appui au plus faible, sans lui donner un maître, elle n'est ni un mal, ni une injustice ». 
La nature positive de la vérité et des connaissances est un appui pour le faible et, plus généralement, un moyen de défense de l'individu dans la société. Plus les masses sont éclairées par les Lumières, moins elles sont enclines à se laisser gouverner par des lois défavorables. L'instruction est le remède le plus efficace contre tout pouvoir injuste, et la vérité apparaît comme ennemie du pouvoir de ceux qui trompent. Condorcet (179I : 261-262) affirme ainsi à plusieurs reprises que le savoir, les connaissances justes, s'opposent naturellement à la mauvaise administration, aux lois injustes : «Plus les hommes seront éclairés, moins ceux qui ont l'autorité pourront en abuser, et moins aussi il sera nécessaire de donner aux pouvoirs sociaux d'étendue ou d'énergie. La vérité est donc à la fois l'ennemie du pouvoir comme de ceux qui l'exercent, plus elle se répand, moins ceux-ci peuvent espérer de tromper les hommes; plus elle acquiert de force, moins les sociétés ont besoin d'être gouvernées ॥. II y a donc un lien étroit entre un régime de vérité scientifique et une administration juste, et réduite. Plus les hommes se comportent en conséquence d'un régime de vérité, moins il est possible de leur appliquer des lois arbitraires. Mais aussi, comme cela apparaît dans cette citation, moins cela est nécessaire car l'organisation humaine émerge de la rationalité des individus. La vérité est l'ennemie du pouvoir, précisément parce qu'elle constitue elle-même un pouvoir, le plus juste qui soit. Le régime de vérité est coercitif mais, contrairement au pouvoir social, il ne s'appuie pas sur un appareil répressif, et opère naturellement.

Puisque la vérité est un pouvoir, il faut se garder de l'instrumentaliser politiquement. Toutefois, bien que Condorcet (179| :258) reconnaisse une difficulté évidente à déterminer l'opinion juste en matière sociale, il souhaite modeler les sciences morales et politiques sur les sciences physiques, munies de vérités démontrées, susceptibles d'être appliquées à tous : « II faut encore chercher à réduire ces sciences à des vérités positives, appuyées, comme celles de la physique, sur des faits généraux et sur des raisonnements rigoureux ; écarter tout ce qui, en parlant à l'âme ou à l'imagination, séduit ou égare la raison, et prouver les vérités avant de prétendre à les faire aimer ». S'il n'est donc pas question de prendre en charge toutes les préoccupations individuelles, la science doit néanmoins permettre de préserver le corps social et de faire en sorte que les citoyens remplissent leur rôle dans la société : « Une éducation générale est préparée pour tous les citoyens; ils y apprennent tout ce qu'il leur importe de savoir pour jouir de la plénitude de leurs droits, conserver, dans leurs actions privées, une volonté indépendante de la raison d'autrui, et remplir toutes les fonctions communes de la société » (Condorcet, I791 :255, mis en italique par nous). Le régime de vérité imaginé par Condorcet permet de concilier l'autonomie individuelle et l'intégrité du corps social. La science du social apparaît alors comme la solution au risque d'éclatement encouru par une société démocratique guettée par l'individualisme. La vérité est un instrument de gestion de la multitude dont personne ne tient le manche (Foucault, 197I). 
Ainsi y a-t-il chez Condorcet une tension permanente entre, d'un côté, l'administration raisonnable permise par des lois justes reposant sur un savoir juste (préservant l'autonomie individuelle) et, de l'autre, l'absence de normes permise par un processus de civilisation issu de l'instruction (préservant l'intégrité sociale) : plus les masses détiennent de connaissances, moins il est possible de leur infliger des règlements injustes, et moins il est nécessaire de les administrer, car la connaissance entraîne l'amour du bien public et une forme d'autorégulation. La révolution n'est donc pas celle d'une forme de gouvernement, mais celle des volontés et des esprits. Dans un tel système, une politique authentiquement rationnelle doit être soumise aux principes de la philosophie, et le projet prend des accents d'utopie platonicienne :

\footnotetext{
«Le seul souverain des peuples libres, la vérité, dont les hommes de génie sont les ministres, étendra sur l'univers entier sa douce et irrésistible puissance ; par elle tous les hommes apprendront ce qu'ils doivent vouloir pour leur bonheur, et ils ne voudront plus que le bien commun de tous. Aussi, cette révolution n'est-elle pas celle d'un gouvernement, c'est celle des opinions et des volontés; ce n'est pas le trône d'un despote qu'elle renverse, c'est celui de l'erreur et de la servitude volontaires; ce n'est point un peuple qui a brisé ses fers, ce sont les amis de la raison, chez tous les peuples, qui ont remporté une grande victoire : présage assuré d'un triomphe universel » (Condorcet, 1791 :272).
}

Ce texte fait de la vérité un instrument de défense contre un pouvoir indissolublement discursif et coercitif, et lie le sort de la question épistémique à celui de la question pratique de l'organisation sociale. Cependant, malgré le désir de faire du régime de vérité scientifique un appui politique de premier ordre, Condorcet reste proche des conceptions baconiennes et, sous maints aspects, la science est encore pensée indépendamment de la société (Pons, 1998). Pour notre réflexion, sa pensée présente l'intérêt de faire d'un système de production du savoir un système de production d'organisation sociale. En effet, le discours de vérité est doté d'une ouverture pratique ainsi que de pouvoirs émancipateurs et coercitifs. Mais la spécificité discursive du pouvoir scientifique, son mode opératoire et l'opération de clôture épistémique ne sont pas encore établis. Avec les auteurs suivants, ces aspects vont être progressivement affinés.

\section{Comte : le positivisme entre théorie et pratique}

Pour Comte, c'est l'introduction du Plan des travaux scientifiques nécessaires pour réorganiser la société (1822) qui a retenu notre attention. Le problème initial du philosophe est politique : il s'agit de rétablir un ordre social acceptable, alors que ni les principes critiques de la révolution, ni ceux, réactionnaires, de la restauration, ne peuvent être institués raisonnablement. La réaction n'est plus en phase avec la 
réalité, et le mouvement révolutionnaire, par sa négation de tout ordre, ne peut mener qu'à une maladie sociale (Blanckaert, 2004). « En un mot, les rois sont en contradiction avec les faits, et les peuples le sont avec les principes » (Comte, I822: 6I). Pour Comte, les anciens principes sont tombés, car ils n'étaient plus en adéquation aux faits, mais il n'y en a pas encore de nouveaux pour les remplacer. D'où une tension entre la loi rétrograde des rois qui va à l'encontre du réel et de l'Histoire, et la doctrine critique des peuples qui ne permet pas de fonder des lois pour la société car elle se résume à la pure destruction. Pour lutter à la fois contre l'arbitraire des rois et contre celui des peuples, il propose de trouver des lois et des règlements fondés sur des vérités positives. On retrouve donc chez Comte la préoccupation de Condorcet pour un ordre social fondé sur un discours doté d'une relation privilégiée avec le réel.

À partir de ce problème d'organisation sociale, la réflexion conduite par Comte aborde de façon originale la diffusion des idées et du savoir. Les savoirs sont pensés comme des doctrines que la société serait apte à recevoir ou non, pour différentes raisons : il y a donc chez Comte un certain relativisme, qui se traduit par une attention soutenue et pragmatique à l'insertion des savoirs dans le contexte social (Grange, 2000). II réfléchit à une doctrine recevable socialement, qui consisterait à la fois en un dépassement et une synthèse des deux qui lui précèdent.Ainsi le savant conçu par Comte doit-il produire simultanément un savoir vrai et un savoir acceptable. Bien entendu, l'acceptabilité du savoir reste fort éloignée d'une vision constructiviste de la production scientifique, mais il est notable que, contrairement aux idées stéréotypées répandues sur le « positivisme comtien », l'auteur tente de produire, plutôt qu'un savoir qui soit la pure expression de la vérité, une pensée toujours consciente d'elle-même et de son inscription dans le monde social. Tout savoir doit rassembler un consensus suffisant pour être actif : « En observant avec précision l'état actuel des nations les plus avancées, il est impossible de n'être point frappé de ce fait singulier et presque contradictoire : quoiqu'il n'existe encore d'autres idées politiques que celles qui se rapportent à la doctrine rétrograde ou à la doctrine critique, aucune des deux, cependant, ne possède plus aujourd'hui, soit chez les rois, soit chez les peuples, une prépondérance véritable; aucune n'exerce une action assez puissante pour diriger la société » (Comte, I 822 : 68). Comme ni le savoir révolutionnaire, ni le savoir réactionnaire ne parviennent à diffuser suffisamment dans la société pour produire une forme d'organisation cohérente, et que l'un et l'autre ont tendance à annuler leurs effets, Comte soutient que les esprits sont prêts à accueillir une nouvelle doctrine : la doctrine positive. À partir d'une pensée politique, en termes d'organisation sociale, il élabore donc un système dont la puissance est cognitive, et s'interprète en particulier par sa circulation sociale. 
Mais avant de rétablir une organisation sociale acceptable, et toujours avec le souci de produire un savoir socialement effectif, il faut légitimer le lieu de savoir qui permette de produire la pensée de cette organisation. Il est donc nécessaire de lutter contre l'état anarchique des intelligences, et de rétablir une hiérarchie savoir-pouvoir. Si chacun prétend savoir, il est inutile que les individus compétents cherchent à déterminer le système juste :

« La doctrine critique a produit dans la plupart des têtes et tend à fortifier de plus en plus l'habitude de s'établir juge suprême des idées politiques générales. Cet état anarchique des intelligences, érigé en principe fondamental, est un obstacle évident à la réorganisation de la société. Ce serait donc vainement que des capacités réellement compétentes formeraient la vraie doctrine organique destinée à terminer la crise actuelle, si, par leur situation antécédente, elles ne possédaient, de fait, le pouvoir reconnu de faire autorité » (Comte, 1822 : 88).

Le système de production de la doctrine positive de l'organisation sociale nécessite donc la création d'un lieu doté d'une légitimité particulière, et le rétablissement d'un ordre des savoirs. Comte en arrive alors à la conception de la clôture épistémique : là où la supériorité du discours vrai relève presque d'une évidence pour Condorcet, qui par conséquent n'en dit mot, Comte pense sa fragilitét. S'il ne fait aucun doute que le discours vrai est supérieur dans l'absolu, il apparait nécessaire de lui ménager un espace de légitimité a priori, qui permettra d'affirmer ses vérités sans affronter de contradiction, et d'établir la base d'un consensus social. Pour ménager cet espace, Comte ( 822 : 83) en appelle à « l'absolue nécessité de séparer les travaux théoriques de la réorganisation sociale prescrite à l'époque actuelle, d'avec les travaux pratiques ». Cette séparation entre théorie et pratique se double d'une attribution précise des rôles: « Dans le système à constituer, le pouvoir spirituel sera entre les mains des savants, et le pouvoir temporel appartiendra aux chefs des travaux industriels » (Comte, 1822 : 88).

Dans cette attribution des rôles, il est cependant nécessaire de noter que la distinction spirituel/temporel ne reconduit pas exactement l'opposition séculaire effectuée par le discours religieux. Car s'il y a bien une dimension métaphysique ou religieuse dans cette conception du pouvoir des savants, il faut aussi entendre le terme «spirituel 》 au sens premier : pouvoir lié à l'esprit, à la pensée (Karsenti, 2006). De cette façon, il ne s'agit pas seulement de créer un « lieu » du savoir légitime, un partage des pouvoirs, et de rétablir une hiérarchie des intelligences : l'objectif de Comte n'est

6 Cette affirmation est à nuancer: Condorcet aussi est soucieux de mettre fin à l'anarchie qui menace la société, au moyen d'un pouvoir scientifique.Voir Baker (1975), en particulier, le chapitre $5:$ « La politique de la science sociale ». 
pas uniquement de mettre les savants à la tête de l'organisation sociale, comme Saint-Simon a pu le rêver pour les industriels. Le rétablissement de la dualité spirituel/temporel et de la hiérarchie qui la commande, au sens religieux, n'est qu'un préalable, un obstacle dont le franchissement est absolument nécessaire à la production d'une nouvelle doctrine organisatrice.

C'est alors à un second niveau qu'il faut comprendre la distinction spirituel/temporel, qui est celui de l'effectivité du pouvoir spirituel. Car l'enjeu se trouve dans le pouvoir du savoir produit sur l'esprit et l'opinion. Sur ce point, nous nous référons à l'analyse de Bruno Karsenti (2006: 50), pour qui le problème du positivisme n'est pas exactement celui d'une politique scientifique : « La sociologie n'achève pas la grande révolution en ce qu'elle dicte enfin au politique ce qu'il doit faire, mais en ce qu'elle fait apparaître, ou plutôt réapparaître, une autre dimension de la politique, résolument mise à l'écart : non pas tant la direction des actions, l'activité qui consiste à légiférer dans l'ordre des phénomènes humains, que la direction des pensées, ce qu'on a appelé justement une politique de l'esprit ». La distinction entre spirituel et temporel n'est donc pas qu'une opposition de lieux et de formes de pouvoir, mais avant tout une distinction entre des modes d'action extrêmement différents (pouvoir agissant sur l'esprit/pouvoir agissant sur le corps) dans laquelle le pouvoir spirituel est premier, car il agit plus en amont.

Le geste de clôture effectué par Comte renvoie ainsi à deux dimensions différentes : d'une part, la création d'un lieu et d'une fonction légitimant la production du discours sur le social, et d'autre part la définition du mode d'action de ce discours, de son pouvoir. La science sociale doit parvenir à élaborer un savoir à la fois descriptif et actif, « vrai » et circulant. Le discours sociologique exercerait ainsi un pouvoir sur l'esprit. Pour cela, comme le fait remarquer Bruno Karsenti $(2006: 56)^{7}$, une intervention directe sur la pensée est nécessaire:

«L'enjeu du pouvoir spirituel moderne n'est donc pas seulement de mettre la pensée de la société au pouvoir, en un lieu que l'autre pouvoir n'attaque pas : elle est aussi, et plus essentiellement, de creuser un accès à cette pensée au plan de l'esprit des sujets, dans leur diversité. En cela, on peut dire que le pouvoir spirituel moderne est le problème natif de la sociologie, prise comme théorie de la société qui cherche à imprégner le tissu social et à lui devenir coextensif mentalement ».

7 On trouve une idée similaire dans le parallèle entre théorie et utopie fait parV. Descombes (1978). 
Ce projet de savoir actif « à l'intérieur de la théorie même » nécessite à la fois l'instauration d'un lieu légitime de production du savoir, et la production d'un discours dont la « positivité » garantisse la diffusion et l'appropriation mentale. Le lieu légitime renvoie au monde savant, clôturé épistémiquement, de façon symbolique et matérielle. De cette clôture émerge un discours positif: la positivité n'est pas une propriété intrinsèque du savoir vrai, mais plutôt une propriété relationnelle, du savoir dans sa capacité à entrer en résonance chez les acteurs et à leur fournir des principes acceptables de la juste implication communautaire.

Entre Condorcet et Comte, une première série de glissements a lieu. Comte approfondit l'analyse du mouvement d'ouverture pratique et définit plusieurs de ses dimensions, afin de produire un savoir acceptable, autour duquel se forme un consensus. Là où Condorcet pose encore ce mouvement dans la naturalité de l'adhésion aux idées justes, Comte examine avec précision les mécanismes de cette adhésion : cela le conduit à la politique de l'esprit mise en lumière par Bruno Karsenti. II établit par ailleurs la nécessité d'une clôture épistémique, qui permettrait de lutter contre l'état anarchique des intelligences, et précéderait l'ouverture pratique de la sociologie. S'il ne fait aucun doute pour Comte que la vérité est entre les mains des savants, il cherche à établir le dispositif permettant d'établir un consensus autour de leur doctrine, en luttant contre l'anarchie sociale.

« Dispositif » est ici à comprendre dans une acception assez différente de celle que lui donnent Michel Foucault (1977) ou, plus récemment, Giorgio Agamben (2006). Plutôt qu'un tissage entre des savoirs, des institutions, des normes dans lesquelles seraient enchâssées les pratiques humaines, nous considérons le dispositif comme la rencontre entre des modes de communication et des opérations réflexives sur ces modes, leur attribuant un certain pouvoir de réalisation. Le dispositif, c'est le discours réflexif qui sélectionne dans le réel, dans la matérialité, un certain nombre d'éléments qu'il rend signifiants et auxquels il prête un pouvoir de réalisation, d'effectuation. Par exemple, le brigand qui menace les voyageurs de la diligence pour leur voler leur argent en expliquant « Personne ne résiste à mon pistolet » produit un dispositif par lequel il attribue à son pistolet (entre autres éléments) le pouvoir d'obtenir l'argent des passagers. De même, le marabout auquel une société prête des pouvoirs est pris dans un dispositif où certaines formes de communication du marabout sont considérées comme performatives. Enfin, dans le cas de la sociologie naissante, il y a production d'un dispositif dans la mesure où toute une réflexion est mise en place sur la possibilité d'élaborer un discours scientifique vrai et acceptable, donc actif. Le dispositif sociologique pensé par Comte ou - comme on le verra - Durkheim met en évidence certaines caractéristiques du discours sociologiques qu'il présente comme potentiellement performatives : en cela il y a production d'un dispositif. 
Comte réalise une part du dispositif en faisant de la séparation des « lieux 》 un élément important de la production du savoir et de son effectivité. Pour Condorcet comme pour Comte, la science sociale est fondée sur la croyance en la possibilité d'établir un ordre social rationnel, et en meilleur accord avec la réalité. Mais Comte sent la nécessité de produire la légitimité sociale du savoir en même temps que celui-ci : c'est pourquoi il pense le rapport entre clôture épistémique et ouverture pratique, posant ainsi les fondements du dispositif durkheimien. Cependant, le rôle central est toujours tenu par l'ouverture pratique, car le discours savant positif agit au niveau des acteurs en s'autovalidant, par l'effet d'une vertu encore peu questionnée. Durkheim renversera le rapport entre ouverture pratique et clôture épistémique, et donnera ainsi au dispositif que nous étudions sa forme caractéristique.

\section{Durkheim et la scientificisation de la sociologie}

Avec Durkheim et l'avènement d'une sociologie scientifique, le discours sociologique connait une seconde série de glissements, comme nous allons le voir. N'hésitant pas à discuter les grands systèmes élaborés par Comte ou Saint-Simon ${ }^{8}$, il défend une conception étroitement scientifique de la sociologie. La discipline qu'il propose n'a pas l'ambition, contrairement à celle de Comte, de déterminer des lois générales du fonctionnement social, mais elle progressera pas à pas en s'appliquant à l'étude de phénomènes concrets. Durkheim peut ainsi être perçu, d'une part, comme l'artisan d'une scientificisation de la sociologie, au prix d'ambitions plus modestes, et d'autre part, comme le défenseur le plus ambigu du pouvoir de la discipline : l'opération de clôture épistémique va cesser d'être un simple préalable à l'ouverture pratique de la science sociale, pour en devenir le fondement.

Cette opération prend d'abord la forme d'un travail explicatif modeste, et surtout du désintéressement sociologique, de la connaissance pour elle-même, garantissant un accès plus solide à la vérité des faits. Une précaution qui apparaît par exemple dans la distinction qu'il fait entre art et science : « La science étudie les faits uniquement pour les connaître et en se désintéressant des applications auxquelles peuvent se prêter les notions qu'elle élabore. L'art, au contraire, ne les considère que pour savoir ce qu'il y a lieu d'en faire, à quelles fins utiles ils peuvent être employés, quels effets nuisibles il faut les empêcher de produire et par quelle voie l'un ou l'autre résultat peut être atteint » (Durkheim, |888-19|4: | |2). L'art élabore des théories, mais elles ne sont pas son

8 Si la filiation de Durkheim avec ces auteurs est certaine, elle procède d'un rapport de rupture et de relectures instructif pour l'analyse (Berthelot, 2002). 
« but immédiat », et elles présentent inévitablement un caractère hâtif et sommaire. Au contraire, « la science n'apparaît que quand l'esprit, faisant abstraction de toute préoccupation pratique, aborde les choses à seule fin de se les représenter » (Durkheim, | 888-19|4 : | |3). || y aurait donc dans la science une ambition descriptive et explicative dénuée de conséquences, et elle serait une activité de représentation, sans intention politique immédiate?.

Mais l'insistance de Durkheim sur la rigueur scientifique de la sociologie a son pendant et ses conséquences du côté de l'action. La sociologie tire de son désintéressement les possibilités d'une action adaptée, et c'est toute l'ambiguïté de ses revendications à la scientificité :

« Celle-ci, comme toute science, étudie ce qui est et ce qui a été, en cherche les lois, mais se désintéresse de l'avenir. [...] Les difficultés pratiques ne peuvent être tranchées définitivement que par la pratique, par l'expérience de chaque jour. Ce n'est pas un conseil de sociologistes, ce seront les sociétés elles-mêmes qui trouveront la solution. Mais il ne peut y avoir que profit à ce qu'un homme au courant des résultats de la science $[\ldots]$ applique sa réflexion à ces matières, et c'est pourquoi les hommes d'action n'auront pas moins d'intérêt à lire [le livre de sociologie de M. de Ferneuil] que les hommes de science » (Durkheim, I888$1914: 225)$.

En effet, bien que le discours sociologique opère une complète clôture épistémique avec Durkheim (production d'un savoir qui aurait un lien privilégié avec le réel), il ne perd pas pour autant toute ambition pratique ${ }^{10}$. Et si la sociologie n'est pas un art, et ne peut trancher les difficultés pratiques de chaque jour, néanmoins « il ne peut y avoir que profit à ce qu'un homme au courant des résultats de la science applique sa réflexion à ces matières ». C'est alors que le véritable projet de la sociologie durkheimienne se dévoile: la sociologie doit restaurer l'unité organique de la société :

« II faut que notre société reprenne conscience de son unité organique ; que l'individu sente cette masse sociale qui l'enveloppe et le pénètre, qu'il la sente toujours présente et agissante, et que ce sentiment règle toujours sa conduite ; car ce n'est pas assez qu'il ne s'en inspire que de temps en temps dans des circonstances particulièrement critiques. Eh bien ! Messieurs, je crois que la sociologie est, plus que toute autre science, en état de restaurer ces idées » (Durkheim, |888-1914: 109-|10).

9 Cependant, la sociologie est à la fois une contrepartie et un aboutissement de la science ${ }_{10}$ politique (Filloux, 1977).

Les théories durkheimiennes de la démocratie sont même tout à fait normatives (Muller, 1993). 
Le rôle politique de la sociologie est essentiel, et celle-ci n'a rien de la connaissance en soi pour laquelle elle se donne. Mais l'action de celle-ci doit être conçue comme une action par le savoir, et repose uniquement sur une élaboration scientifique du savoir. Pour éclairer l'opinion, la science remet sans cesse en question les limites de son propre domaine, et acquérir toujours plus de scientificité.

Comme Comte, Durkheim porte le projet d'un savoir qui agirait de l'intérieur, qui modifierait les comportements et produirait l'organisation qui manque à sa société. Mais pour Durkheim, la dimension normative du projet émerge d'elle-même, de sa propre scientificité. D'où, par exemple, son intention de traiter scientifiquement la morale : au lieu de dire ce que doivent être les institutions, il faut dire ce qu'elles sont. Une ambiguïté persiste cependant : un pur travail descriptif est-il envisageable?

\footnotetext{
« La morale est même de toutes les parties de la sociologie celle qui nous attire de préférence et nous retiendra tout d'abord. Seulement, nous essayerons de la traiter scientifiquement. Au lieu de la construire d'après notre idéal personnel, nous l'observerons comme un système de phénomènes naturels que nous soumettrons à l'analyse et dont nous chercherons les causes : l'expérience nous apprendra qu'elles sont d'ordre social. Sans doute nous ne nous interdirons pas toute spéculation sur l'avenir, mais n'est-il pas clair qu'avant de chercher ce que doivent être la famille, la propriété, la société, il faut savoir ce qu'elles sont, à quels besoins elles correspondent, à quelles conditions elles doivent se conformer pour vivre ? C'est par là que nous commencerons et par là se résoudra d'elle-même une antinomie qui n'est pas sans avoir douloureusement troublé les consciences » (Durkheim, |888-1914: 106).
}

Le projet scientifique de la sociologie arrive ainsi à son apogée, et devient le fondement de son ouverture pratique. Durkheim renverse alors le rapport entre clôture épistémique et ouverture pratique, et donne sa forme achevée à la fiction du décalage entre production de discours et production d'actions : la quête de la scientificité du savoir devient la condition de son opérationnalité.

\section{Conclusions partielles}

Pierre Bourdieu (1980:48, in : Rancière, 1984: 355) interprète l'établissement de ce rapport comme une stratégie : « La sociologie est, dès l'origine, dans son origine même, une science ambiguë, double, masquée : qui a dû se faire oublier, se nier, se renier comme science politique pour se faire accepter comme science universitaire ». L'efficacité politique de la sociologie serait ainsi fonction de sa légitimité universitaire. Jacques Rancière (1984: 355) s'oppose à cette conception, qui selon lui ne tient « que par le recours aux concepts désincarnés de la tour d'ivoire universitaire et de l'engagement politique ». Bien au contraire, 
pour Jacques Rancière, la III République est par excellence le lieu de la croyance en une Université militante, qui par la science joue un rôle politique. II n'y aurait donc nulle stratégie, nul oubli dans la démarche de Durkheim, mais plutôt une éthique du savoir qui affirmerait la science comme outil politique essentiel. Faut-il donc y voir avec Pierre Bourdieu une stratégie? Avec Jacques Rancière la posture éthique d'une époque? Ou encore une croyance nécessaire au déroulement de l'activité scientifique (Rorty, 1982)?

Plutôt que de trancher entre ces interprétations, nous préférons observer le fonctionnement concret de ce rapport, et ses conséquences. Toujours à la recherche d'un lieu d'où il pourrait affirmer la pureté de son discours, le sociologue ne cesse de s'abstraire du social par diverses médiations. Mais cette tentative toujours réitérée de purifier son discours et de lui donner un rapport privilégié avec les « faits » ne va pas sans l'intention de le poser au principe de tout comportement social. II est évidemment impossible de produire le lieu « hors-lieu » à partir duquel jaillirait le savoir sur le monde social. Les médiations introduites ne règlent en rien l'appartenance profonde des sciences sociales au monde social. Le rapport ouverture/fermeture du discours sociologique conduit alors, dans le sillage durkheimien, la sociologie à passer sous silence un aspect essentiel du dispositif qu'elle constitue : la diffusion sociale du savoir. Afin de démontrer cet aspect, nous reviendrons sur la façon dont ce double rapport au savoir a provoqué une conception particulière de la communication des savoirs, et en particulier une prétention à résoudre la polyphonie sociale.

\section{Polyphonie sociale}

\section{Un savoir sans médiation?}

Le rapport entre clôture épistémique et ouverture pratique, qui aboutit chez Durkheim comme rapport de fondement, est lourd de conséquences sur le plan communicationnel ; il est indissociable d'un dispositif dont la communication est absente. Avec Durkheim, la sociologie règle la question communicationnelle de la circulation sociale des savoirs : la diffusion est subordonnée à la production d'un discours clos, abstrait des connaissances communes, et c'est la rationalité de la connaissance sociologique qui assurera sa diffusion sociale. II n'est donc plus nécessaire de s'interroger sur les médiations concrètes qui affectent la diffusion des savoirs, puisque leur circulation est soumise à leur rationalité. Par exemple, Bernard Lahire propose d'enseigner « rationnellement »les sciences sociales dès l'école primaire, mais si sa proposition mérite d'être discutée, 
elle ne porte aucune trace de réflexion sur la façon dont des élèves s'approprient ce savoir, sans doute fort différente entre des élèves du primaire et des étudiants à l'université. II s'agit évidemment dans son cas d'un texte conclusif ouvert et volontairement utopique, mais néanmoins révélateur d'une manière de régler la question communicationnelle dont nous avons mis les jalons en évidence.

Le dispositif spécifique mis en place avec Durkheim peut donc être interprété comme une tentative de régler le problème communicationnel, de produire un discours indépendant des médiations. Pourtant, c'est en circulant dans la société, en faisant l'objet de médiations, qu'un discours acquiert un degré de validité pour les acteurs. Par exemple, les discours d'institutions traditionnelles de socialisation tirent leur valeur de la façon dont ils permettent aux acteurs de rendre leurs pratiques cohérentes. De la même façon, le projet sociologique ne tient pas seulement grâce au dispositif de clôture épistémique et d'ouverture pratique : par exemple, l'école durkheimienne met en place de nombreuses stratégies de diffusion du paradigme sociologique dans des champs très variés, notamment par le biais de L'Année sociologique (Mucchielli, 1998). Nulle clôture épistémique, nulle coupure épistémologique n'opèrent alors : les discours tirent, sinon leur rationalité, du moins leurs raisons, des pratiques dans lesquelles ils s'inscrivent et auxquelles ils donnent sens. Si n'importe quel discours (idéologique, traditionnel, scientifique, etc.) peut «fonctionner », c'est parce qu'il relaye et est relayé par des pratiques" ". I y a donc simultanéité entre la construction des discours et leur enrôlement dans les pratiques, et non un rapport de fondement tel que le rêverait pour la science la sociologie durkheimienne.

Dès lors, la distinction opérée dans le sillage durkheimien entre un plan discursif, qu'il faudrait protéger autant que possible des assauts du sens commun, et un plan pratique, qui en dépendrait, ne permet pas de rendre compte de tous les phénomènes sociaux. Sa valeur heuristique est indéniable, mais elle conduit en particulier à fermer les yeux sur les mécanismes de la circulation des savoirs, et à produire le mythe d'un savoir capable de mettre fin au bruit social, et à la polyphonie.

\section{La polyphonie sociale}

Le mythe assumé par la sociologie avec Durkheim est celui d'un savoir qui permettrait d'organiser extérieurement un monde parcouru par une infinité de voix, et condamné à la polyphonie sociale : en distinguant, comme nous l'avons vu, le plan épistémique du plan pratique, et en fondant la possibilité

\footnotetext{
"I Sur l'« impossible coupure », voir Boure (1997).
} 
du second sur la clôture du premier, le discours sociologique s'interdit de penser son inscription dans cette polyphonie. Chez Comte et Durkheim, le moment fondateur du discours sociologique est son abstraction du monde social. C'est à partir de ce moment que la sociologie balaiera les prétentions des autres discours sociaux à la validité, et fondera l'action individuelle et collective sur ses propres principes. Dans une telle situation, les acteurs s'approprieraient un discours sans concurrents, et l'action juste serait permise par la résolution de la polyphonie sociale.

Mais les années 70 et 80 ont montré, notamment avec le retour des acteurs dans l'analyse, que le fantasme sociologique devait être retourné et qu'il s'agissait de « rendre la parole » plus que de «parler pour ». L'évolution du travail d'un auteur comme Pierre Bourdieu (1993) dans La misère du monde est tout à fait révélatrice sur ce point :il ne s'agit plus tant de mettre au jour les structures qui déterminent les « agents » que de médiatiser leur parole. Ce tournant, négocié par des auteurs comme Alain Touraine puis Luc Boltanski ou Bruno Latour, est fort bien analysé par François Dosse (1997). Nous ne pouvons que souligner ici l'importance qu'il aura dans la détermination de l'existence du phénomène polyphonique, qui nous intéresse à présent. Pour définir la polyphonie sociale, nous élargissons la notion développée parYves Jeanneret (2005), aux mécanismes sociaux. Chez ce chercheur, la notion de polyphonie s'applique au matériau du texte de science sociale :

\footnotetext{
«Rendre compte d'un «terrain», défendre une «théorie», c'est dire quelque chose de ce qui est censé structurer les pratiques des autres. Prétendre établir une «loi», c'est énoncer ce qui s'impose à tous. Inversement, dénoncer les positions de surplomb ou revendiquer des concepts indigènes, c'est soumettre la recherche au discours ordinaire. Dans tous les cas, faire science, c'est écrire au pluriel. C'est rédiger une monographie polyphonique, acte scripturaire nourri d'une pluralité de voix » (Jeanneret, 2005 : 59).
}

II fait de cette caractéristique le point nodal de la production de savoir en sciences sociales, car il s'agit de dépasser la pluralité des discours pour produire un texte cohérent et unifié. La définition de la polyphonie que nous mobilisons ici ne concerne cependant pas la seule nature du texte de science sociale, mais aussi le milieu dans lequel s'inscrit le savoir des sciences sociales. Tout texte de science sociale opère une gestion de la polyphonie, et simultanément s'inscrit dans un contexte polyphonique : il ne peut échapper au monde qui l'entoure.

Malgré tout, la sociologie propose avec Durkheim de mettre fin au dissensus et à la polyphonie par la production d'un régime de vérité. Apparaît alors une relation directe entre le mythe d'un discours capable de mettre en adéquation la parole et les actes, et le fantasme de la fondation d'un ordre social rationnel : la multiplicité des discours sociaux n'est plus un objet de pensée si le problème fondamental de l'organisation sociale 
est réglé. Mais pas plus qu'il n'existe une « vérité » sociale, le sociologue n'a pas le pouvoir théorique de résoudre pratiquement les tensions qui parcourent la société. Puisqu'il n'y a ni « hors-société », ni savoir « vrai » sur la société, le sociologue ne peut définitivement mettre fin au régime polyphonique dans lequel il ne fait qu'inscrire son propre discours. On ne peut donc passer sous silence la pluralité des discours à laquelle se confronte le savoir des sciences sociales. Pour Baudouin Jurdant (1999: 150), on ne peut ignorer l'existence d'autres voix:

"Côté "société", [...] les faits parlent effectivement d'eux-mêmes. lls ont toujours déjà du sens. Et c'est souvent contre ce bavardage encombrant des faits sociaux, que les scientigrecs [praticiens de sciences sociales] doivent lutter pour imposer une nouvelle vision des faits [...]. Impossible, évidemment, pour ces scientigrecs d'ignorer que cette nouvelle vision qu'ils proposent vient d'eux-mêmes et de la manière dont ils ont intériorisé un certain idéal de science. Le regard qu'ils portent sur les phénomènes sociaux doit précisément son originalité à l'intériorisation de cet idéal. Mais il ne peut ignorer qu'il n'est lui-même qu'un regard parmi d'autres possibles, tout prêts à défendre leur légitimité. Un tel manque de lucidité serait non seulement épistémologiquement absurde (nul ne peut occuper un poste d'observation de la réalité sociale dégagé de cette réalité), mais encore éthiquement condamnable (pluralité et relativité des points de vue sont nécessaires au maintien des libertés individuelles) ».

Le jugement porté par Baudouin Jurdant montre toute l'importance qu'il y a à prendre en considération l'existence d'autres « regards » : ne pas le faire serait épistémologiquement absurde, éthiquement condamnable, et on pourrait ajouter « politiquement inconséquent », puisque cela reviendrait à nier l'implication politique de la recherche en science sociale.

\section{Implications politiques}

L'ignorance dans laquelle la science sociale tient les mécanismes de la polyphonie la conduit à réduire la dimension politique fondamentale de la circulation des savoirs, à leur diffusion. Nous avons survolé l'élaboration de cette conception, chez Condorcet, Comte et Durkheim, et nous avons montré comment, en jetant un voile pudique sur le rapport du savoir sociologiqueàl'action,Durkheima surmontéla question communicationnelle. Mais nous aurions aussi bien pu remonter beaucoup plus loin : chez Platon, par exemple, le projet de république s'imposera de lui-même ou ne sera pas. Les citoyens devraient s'y plier de plein gré, s'y abandonner. La science sociale est alors la science qui met fin au politique : qui le conduit à ses fins, qui l'achève. De ce point de vue, la théorie marxiste représente un accomplissement, comme l'a montré Régis Debray. Marx, plus encore que 
Durkheim, fait preuve d'une foi inébranlable dans le savoir vrai comme potentiel de transformation de la société, et il prête peu d'attention aux mécanismes de diffusion et d'appropriation de son œuvre. Il est ainsi amené, contrairement à ce que l'on pourrait croire, à dissoudre le politique dans la science. Régis Debray (1980:62) note : « La politique, l'art des médiations, l'artifice du malheur humain, Marx ne promet pas seulement son abolition, il entend démontrer qu'un jour elle ne sera plus nécessaire ». Pour Marx, il n'y a en effet nul besoin de politique, dans la mesure où l'acte politique est précisément cet acte de médiation que la science économique et sociale ignore, remettant la transformation de la société au pouvoir de vérité du savoir scientifique.

Le mythe d'une rationalité politique retire toute nécessité à des mécanismes de diffusion et de médiation : l'ordre surgit de lui-même, et de la diffusion rationnelle du savoir. Mais si la fortune pratique du marxisme ne peut s'expliquer par son contenu théorique, il en va de même pour toutes les sciences sociales, et en particulier en sociologie. C'est donc la politique que nous proposons de réintroduire dans le champ du savoir, avec l'étude de la circulation sociale des savoirs : comprendre comment le savoir circule, c'est tenir compte du mouvement de va-et-vient qu'il effectue dans le champ politique, de ses appropriations et de ses rejets. La politique tient à l'ensemble des mécanismes de qualification d'un savoir : acceptation, rejet, discussion, et c'est à la compréhension de ces mécanismes qu'il faut aspirer. De plus, les enjeux politiques d'aujourd'hui ne se trouvent pas seulement dans la lutte contre les inégalités socio-économiques. Ils sont plus généralement dans l'étude des légitimités, des droits à la parole, dans la maîtrise des médiations et de la circulation des discours. Or, le projet de science sociale participe pleinement à ce mouvement de maîtrise des discours, tout en se refusant d'en analyser les ressorts. Si l'on juge la science sociale à l'aune de son projet politique, il est donc nécessaire d'analyser en profondeur les mécanismes communicationnels qui président à ses réappropriations : les discours ne fonctionnent pas uniquement à un niveau discursif, mais aussi en relayant et en se matérialisant dans des pratiques, et il faut rétablir, plutôt qu'une forme de réflexivité déjà présente dans le discours sociologique, une prise en compte des médiations par lesquelles les discours circulent socialement.

\section{Conclusion}

La société contemporaine a placé dans les savoirs des enjeux capitaux. La lutte des classes a laissé place, si l'on caricature, à une « lutte des savoirs 》. Bien entendu, il ne s'agit pas, pour les sciences sociales, de substituer aux inégalités économiques et sociales, qui persistent effectivement, des inégalités épistémiques, discursives ou symboliques. De même, il serait absurde de voir 
dans les enjeux symboliques une invention récente, alors que ceux-ci ont toujours existé. En revanche, il est nécessaire de tenir compte de la place nouvelle que les débats autour de ce que nous désignons largement sous le terme de « savoirs » occupent dans l'élaboration des régimes de pouvoirs, dans l'établissement de hiérarchies et d'inégalités. En effet, la tertiarisation de l'économie, le rôle croissant des médias, ou encore la marchandisation de la culture contribuent à placer dans les savoirs des enjeux politiques essentiels. Légitimer un discours, attribuer un droit à la parole, définir un partage culturel : autant d'actes qui participent de la construction d'oppositions et d'inégalités sociales. II est donc urgent d'analyser ces formes de partage, de gestion et de mise en circulation des savoirs. Dans ce contexte, les sciences de l'information et de la communication (sic), définies comme étude de la circulation des savoirs, sont amenées à jouer un rôle de premier plan. Elles peuvent donner les moyens de mieux comprendre les enjeux liés aux différents savoirs, à leur construction et à leur confrontation avec d'autres. Ainsi le projet communicationnel apparait-il indissolublement épistémologique et politique, en ce qu'il traite à la fois le procès de formation des savoirs et leur inscription dans le monde social.

En tant que formation discursive, les sciences sociales constituent par ailleurs un objet d'intérêt pour les SIC, sur des problématiques qui jusqu'à présent étaient relativement absentes des préoccupations des chercheurs. En abordant le problème de la tension entre clôture épistémique et ouverture pratique dans les sciences sociales, nous voulons souligner l'importance de la prise en compte du lien indissoluble entre savoir et pratique. Comme nous l'avons dit, pour affermir l'approche épistémologique des sciences sociales, il est primordial de penser les conditions et les modalités de la circulation du savoir des sciences sociales dans la société : sa vulgarisation, et toutes ses formes d'appropriation. Étudier la circulation du savoir des sciences sociales revient à poser différemment notre regard sur ce champ, à éviter certains clichés épistémologiques sur la spécificité disciplinaire, et à penser autrement les possibilités d'action politique offertes par la discipline. Si les sciences sociales cherchent à comprendre la polyphonie sociale, elles recourent pour cela à un traitement particulier de la relation savoir/action, et produisent un savoir qui se trouve ainsi toujours entre aporie et expertise, sans résoudre cette tension fondamentale. Étudier la mise en circulation du savoir des sciences sociales, c'est chercher à résoudre cette tension, et refuser de considérer, comme bien des travaux d'épistémologie, qu'il est alors seulement question de diffusion.

II faut donc interroger inlassablement les mécanismes de diffusion et de circulation, les effets de théorie ou de réalité, ${ }^{12}$ qui sont des problèmes centraux de la pratique scientifique en même temps que de réels enjeux

\footnotetext{
${ }^{12}$ Voir par exemple l'article d'É. Neveu et R. Rieffel (199|).
} 
politiques. II ne s'agit pas seulement de produire un savoir rationnel sur l'homme pour permettre une organisation plus rationnelle de la société, mais aussi de permettre aux acteurs de s'approprier ce savoir. Notre proposition ne se résout donc pas par une simple réflexivité, au sens où le sociologue se prendrait lui-même pour objet. S'il faut penser les conditions d'élaboration et surtout de diffusion et d'appropriation du savoir sociologique, c'est sur un mode indissolublement épistémologique et politique.

\section{Références}

Agamben G., 2006, Qu'est-ce qu'un dispositif ?, trad. de l'italien par M. Rueff, Paris, Éd. Rivages, 2007.

Baker K., 1975, Condorcet, raison et politique, trad. de l'anglais par M. Nobile, Paris, Hermann, 1988.

Berthelot J.-M., 1998, L'intelligence du social, Paris, Presses universitaires de France.

— 2002, « Durkheim, lecteur de Comte? », pp. 1 85-206, in : Bourdeau M., Chazel F., dirs, Auguste Comte et l'idée de science de l'homme, Paris, Éd. L'Harmattan.

Blanckaert C., 2004, La nature de la société, Paris, Éd. L'Harmattan.

Bourdieu P., 1980, Questions de sociologie, Paris, Éd. de Minuit.

— 1993, La misère du monde, Paris, Éd. Le Seuil.

Boure R., 1997, « Les sciences de l'information et de la communication au risque de l'expertise ? », Réseaux, 82-83, pp. 233-253.

Comte A., I 822, Plan des travaux scientifiques nécessaires pour réorganiser la société, Paris, Éd. L'Harmattan, 200I.

Condorcet, 1791, Cinq mémoires sur l'instruction publique, Paris, Flammarion, 1994.

Debray R., 1980, Le scribe, Paris, Grasset.

Descombes V., 1978, «L'utopie positive », pp. I 49- I 89, in : COLLECTIF, Régénération et reconstruction sociale entre 1780 et 1848, Paris, Vrin.

De Singly Fr., 2005, L'individualisme est un humanisme, La Tour-d'Aigues, Éd. de L'Aube.

Dosse Fr., 1997, L'Empire du sens, l'humanisation des sciences humaines, Paris, Éd. La Découverte.

Durkheim É., |888-1914, La science sociale et l'action, Paris, Presses universitaires de France, 1987.

Filloux J.-C., 1977, Durkheim et le socialisme, Genève, Droz.

Foucault M., 197I, L'ordre du discours, Paris, Gallimard.

— 1977, « Le jeu de Michel Foucault » (entretien avec D. Colas et al.), Ornicar?, Bulletin périodique du champ freudien, 10, juil.

Grange J., 2000, Auguste Comte, la politique et la science, Paris, O. Jacob.

Heilbron J., 1990, Naissance de la sociologie, trad. du néerlandais par P. Dirkx, 
Marseille, Agone, 2006.

Jeanneret Y., 2005, « Une monographie polyphonique. Le texte de recherche comme appréhension active du discours d'autrui », Études de communication, 27, févr., pp. 57-74.

Jurdant B., 1999, « Le désir de scientificité », Alliage, 41-42, pp. | 47- 155.

Karsenti B., 2006, Politique de l'esprit, Paris, Hermann.

Kaufmann J.-Cl., 200 I, Ego, Paris, Nathan.

Lahire B., 2002, « Utilité : entre sociologie expérimentale et sociologie sociale », pp. 43-66, in : Lahire B., dir., À quoi sert la sociologie ?, Paris, Éd. La Découverte.

— 2005, L'esprit sociologique, Paris, Éd. La Découverte.

Latour B., 2006, Défaire la société, Paris, Éd. La Découverte.

Licoppe C., 1996, La formation de la pratique scientifique, Paris, Éd. La Découverte.

Mucchielli L., 1998, La découverte du social : naissance de la sociologie en France, Paris, Éd. La Découverte.

Muller H.-P., 1993, « Durkheim's political sociology », pp. 95- 110 in :Turner S., ed., Emile Durkheim, sociologist and moralist, London, Routledge.

Neveu É., Rieffel R., 1991, «Effets de réel dans les sciences de l'information et de la communication », Réseaux, 50, pp. I1-39.

Pons A., 1998, « De la Nouvelle Atlantide au Fragment sur l'Atlantide. Science et société chez Bacon et Condorcet », pp. 170-184, in :Tinland F., dir., Nouvelles sciences, Seyssel, Champ Vallon.

Rancière J., 1984, « L'éthique de la sociologie », pp. 353-376 in : Rancière J., Les scènes du peuple, Paris, Horlieu, 2003.

Rorty R., 1982, « Méthode, science sociale et espoir social », pp. 345-374 in : Rorty R., Conséquences du pragmatisme, trad. de l'anglais (américain) par J-P Cometti, Paris, Éd. Le Seuil, 1993.

Shapin S., Schaffer S., 1989, Léviathan et la pompe à air, trad. de l'anglais par Th. Piélat et S. Barjansky, Paris, Éd. La Découverte, 1993.

Wagner P., 2000, «An entirely new object of consciousness, of volition, of thought », pp. I 32- 157, in : Daston L., ed., Biographies of scientific objects, Chicago, Chicago University. 


\section{$>$ NOTES DE LECTURE}

Cinéma, télévision, images

3II Stéphane Benoist, Anne Daguet-Gagey, dirs, Un discours en images de la condamnation de mémoire (Julien Trapp)

312 Steven Bernas, Montage créatif et processus esthétique d'Eisenstein, suivi de «Montage 38 » d'Eisenstein (Claire Martel)

314 Jérôme Bimbenet, Film et histoire (Marie Louis)

316 Tamara Chaplin, Turning on the mind. French Philosophers on Television (Jérôme Bourdon)

317 Évelyne Cohen, Marie-Françoise Lévy, La télévision des Trente Glorieuses. Culture et politique (Laurence Leveneur)

318 François Dosse, Jean-Michel Frodon, dirs, Gilles Deleuze et les images (Stéphane Olivesi)

320 Jean-Michel Frodon, dir., Le cinéma et la Shoah, un art à l'épreuve de la tragédie du XX siècle (Vincent Lowy)

323 Gilles Lipovetsky, Jean Serroy, L'écran global. Culture-médias et cinéma à l'âge hypermoderne (Gilles Bœnisch)

324 Marie-José Mondzain, Homo Spectator (Steven Bernas)

325 René Prédal, Esthétique de la mise en scène (Frank R. Links)

327 Jean-Claude Soulages, Les rhétoriques télévisuelles. Le formatage du réel (Yeny Serrano)

\section{Genres}

Marie-Joseph Bertini, Femmes : le pouvoir impossible (Marieke Stein)

Tanella Boni, Que vivent les femmes d'Afrique? (Naila Amrous)

330 Sylvain Ferez, Le corps homosexuel en-jeu. Sociologie du sport gay et lesbien (Nelly Quemener)

332 Gérard Neyrand, Abdelhafid Hammouche, Sahra Mekboul, Les mariages forcés. Conflits culturels et réponses sociales (Sylvie Thiéblemont-Dollet)

335 Françoise Thébaud, Écrire l'histoire des femmes et du genre (Catherine Gravet)

337 Josette Trat, Diane Lamoureux, Roland Pfefferkorn, dirs, L'autonomie des femmes en question. Antiféminismes et résistances en Amérique et en Europe (Philippe Hamman) 